\title{
An Algorithm for Fast Calculation of Short Circuit Forces in High Current Busbars of Electric Arc Furnace Transformers Based on Method of Images
}

\author{
Masood Moghaddami ${ }^{\mathrm{a}}$, Amir Moghadasi ${ }^{\mathrm{a}}$, Arif I. Sarwat ${ }^{\mathrm{a} * *}$ \\ ${ }^{a}$ Electrical and Computer Engineering Department, Florida International University, Miami, Florida.
}

\begin{abstract}
This paper proposes an algorithm for the calculation of short circuit forces on the high current busbars of electric arc furnace (EAF) transformers based on the method of images using an analytical solution for the electromagnetic force between two adjacent current carrying rectangular conductor. The theory of images is used to account for the impact of the tank walls on the short circuit forces on the busbars. The proposed method uses an iterative algorithm and increases the number of image layers to achieve the desired accuracy. A 30 MVA EAF transformer is investigated as a case study and the results are compared to 2D finite element analysis (FEA). ANSYS software is used for the FEA. The proposed algorithm converges very fast, so that only in its first iteration, the convergence error is less than $0.2 \%$. The comparisons show that the short circuit force calculations using the proposed method, conform to the 2D FEA results. However, the short-circuit calculation time using the proposed method is about 20 times faster than the 2D FEA with the same relative error. Therefore it can be used as a faster alternative for the FEA. The proposed method is characterized by fast convergence, simple calculations and high precision.
\end{abstract}

Keywords: busbar, electric arc furnace, method of images, power transformer, short circuit force.

1. Introduction

Nowadays, electric arc furnace (EAF) transformers are widely used in smelting industries. The proper design and sufficient production technology of the EAF transformers are fundamental for a high efficiency of the plant operation. Furnace transformers are one of the most valuable apparatuses in electric systems of smelting industries. Any failure will lead to high costs for the repair or replacement and may often lead to very high costs due to temporary loss of the power delivery capability. Therefore, EAF transformers are highly protected against various causes of failure. This type of transformer must

${ }^{*}$ Corresponding author

Email address: asarwat@fiu.edu (Arif I. Sarwat)

This work was funded by the National Science Foundation under grant number CRISP-1541069. 


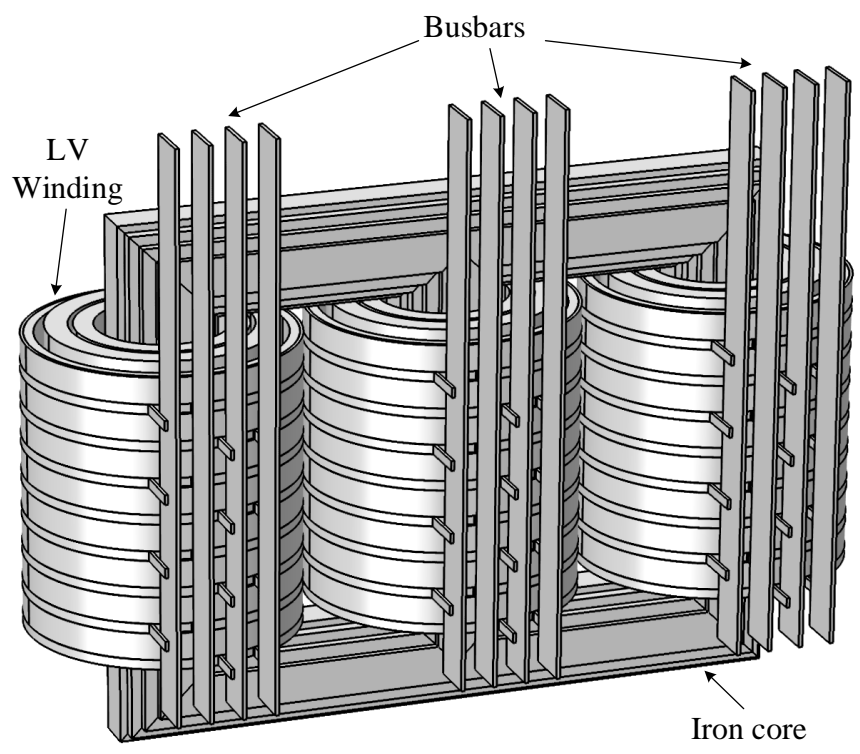

Figure 1: A typical electric arc furnace (EAF) transformer with high-current low-voltage output busbars.

have the capability to withstand the extremely high levels of electromagnetic forces due to short circuit faults. One of the important structural parts of a furnace transformer are high current low voltage output busbars. A typical EAF transformer with low voltage busbars is shown in Figure 1. These busbars are used to transfer the high currents from the low voltage windings disks to the EAF through high current cables. Because of the very high currents in these busbars (e.g. $100 \mathrm{kA}$ ) special attention and design considerations are required [1]-[4]. Also, due to the low short circuit impedance at the low voltage side, the short circuit currents are very high and as a result the electromagnetic forces in busbars are critically high. Different numerical and analytical analysis of general purpose power transformers has been well studied in many publications [6]-[12].

The electromagnetic analysis of EAF transformers has been investigated in [7]-[24]. In [13] and [14], the current distribution in low voltage windings of EAF transformers has been discussed. The electromagnetic forces in high current busbars are investigated in [15] and [16]. In [15], the electromagnetic forces are calculated using 2D finite element analysis (FEA). The short circuit forces of busbars with multiple sub-conductors are calculated based on a correction factor method in [16]. The method of images is a well-known technique which has many applications in electromagnetics and has been widely used in different studies [2],[7],[18],[21]-[23]. Specially, this method can be used for the calculation of different field parameters in power transformers, e.g. leakage field, short circuit forces, eddy current losses, etc. In [7] and [23], the method images is used for the calculation of the leakage field and the electromagnetic forces on the windings of power transformers and the results are compared to 2D FEA. The error associated with the use of the method of images in very low frequencies [22] (e.g. $50 / 60 \mathrm{~Hz}$ 
which our study deals with) or very high frequencies analysis of electrical devices [18] are very low. However, The theory of images in its simple form cannot be used for medium frequencies anaylsis, and may lead to significant error and, the location and magnitudes of the images should be modified so that the boundary conditions in medium frequencies could be satisfied with the images [2]. In [17], the magnetic effects of induced eddy currents in thin conducting sheets and the transient electromagnetic forces are studied by means of moving current images and by using an electrical circuit analogy.

In this paper, an algorithm for fast and accurate calculation of the short circuit forces on highcurrent busbars of EAF transformers is presented. The proposed algorithm applies the theory of images to an analytical solution for the electromagnetic force between adjacent rectangular busbars, to account for the impact of the transformer tanks walls on the short-circuit forces. The analytical solution for the electromagnetic force is verified using 2D FEA. A 30 MVA EAF transformer is investigated as a case study and the results are compared to the corresponding 2D FEA to verify the accuracy of the analytical method. The comparisons show that the proposed algorithm completely conform to the 2D FEA with a much lower calculation time. Also, the short circuit calculations show that the short circuit forces on the high current busbars are of a great strength and therefore, should be considered as an important design measure in EAF transformers.

\section{Analytical Calculation of the Magnetic Field of a Single Busbar}

In order to find an analytical solution for the electromagnetic force between two rectangular busbars, magnetic field distribution of a single rectangular conductor is required. The derivation of the electromagnetic force is described in section 3. The magnetic field of a single rectangular conductor can be calculated analytically by assuming a uniform current density inside the conductor. The cross section of a typical rectangular conductor with the dimensions of $a \times b[m]$ and a uniform current density $J\left[A / m^{2}\right]$ perpendicular to $x-y$ plane is shown in Figure 2. By applying the Biot-Savart law [6] and integrating the magnetic field of the current elements $J d x d y$ over the cross section of the conductor $S_{c}$, the total magnetic field of a rectangular conductor can be calculated using the following equation:

$$
H=\iint_{S_{c}} \frac{J a_{\phi}}{2 \pi\|R\|} d x^{\prime} d y^{\prime}
$$

where $H[A / m]$ is the magnetic field vector at point $(x, y),\left(x^{\prime}, y^{\prime}\right)$ are the current elements coordinates, $J\left[A / m^{2}\right]$ is the current density of the conductor, $R[m]$ is the vector from $\left(x^{\prime}, y^{\prime}\right)$ to $(x, y), a_{\phi}$ is the unit vector perpendicular to $R$ vector. By converting $R$ and $a_{\phi}$ into Cartesian form, (1) can be rewritten 
as follows:

$$
H=\frac{J}{2 \pi} \int_{y_{c}-\frac{a}{2}}^{y_{c}+\frac{a}{2}} \int_{x_{c}-\frac{b}{2}}^{x_{c}+\frac{b}{2}} \frac{-\left(y-y^{\prime}\right) a_{x}+\left(x-x^{\prime}\right) a_{y}}{\left(x-x^{\prime}\right)^{2}+\left(y-y^{\prime}\right)^{2}} d x^{\prime} d y^{\prime}
$$

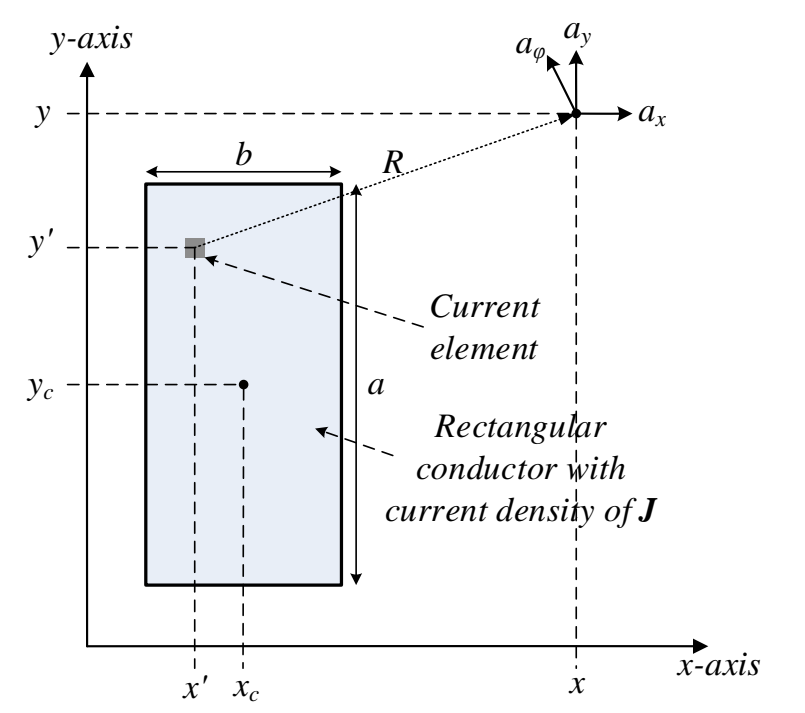

Figure 2: The definition of parameters used for the calculation of magnetic field of rectangular conductor with a uniform current density of $J\left[A / m^{2}\right]$.

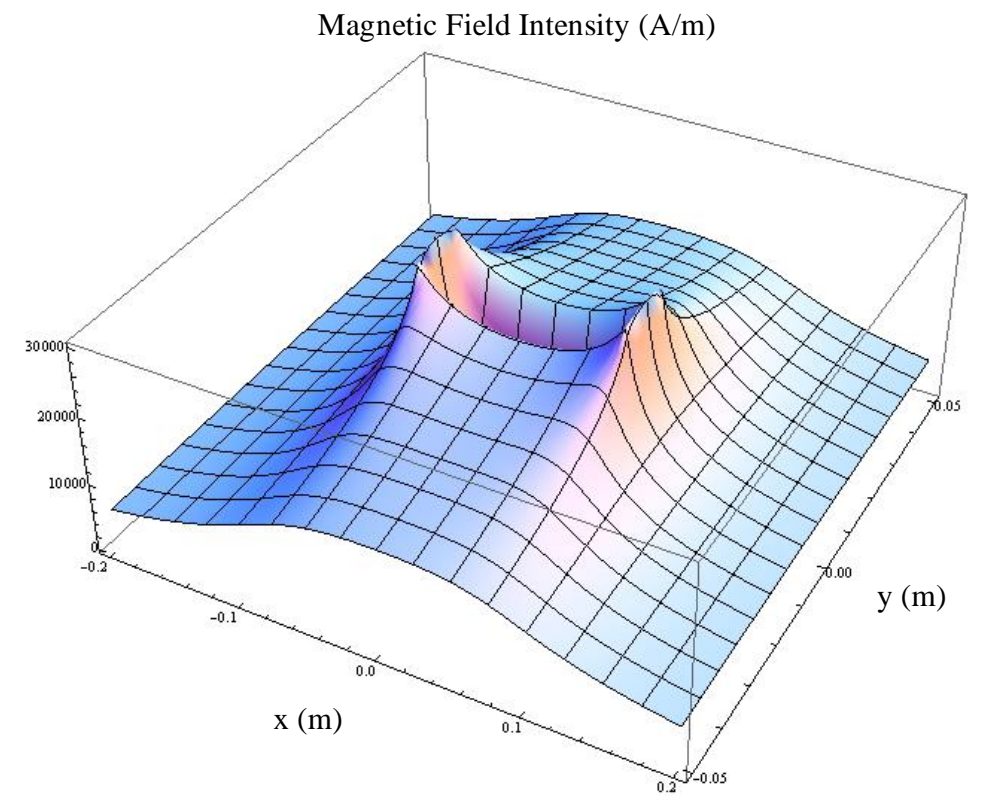

Figure 3: The magnetic field distribution of a single busbar with $7 \mathrm{kA}$ current and dimensions of $150 \mathrm{~mm} \times 20 \mathrm{~mm}$ located at the origin of the coordinate system. 
where $\left(x_{c}, y_{c}\right)$ are the coordinates of the center of the busbar, $(x, y)$ are the coordinates of an arbitrary point at which the magnetic field is desired and $a_{x}$ and $a_{y}$ are unit vectors along $x$ and $y$ axes respectively. By assuming that the center of the conductor is located at the origin of the $x-y$ coordinate system $\left(x_{c}=0\right.$ and $\left.y_{c}=0\right)$, the components of the magnetic field, $H_{x}$ and $H_{y}$ can be calculated as the following set equations:

$$
\begin{aligned}
H_{x}=-\frac{J}{8 \pi}[ & (4 x+2 a)\left(\tan ^{-1}\left(\frac{b+2 x}{a+2 y}\right)+\tan ^{-1}\left(\frac{b-2 x}{a+2 y}\right)\right) \\
& +(4 y-2 a)\left(\tan ^{-1}\left(\frac{b+2 x}{a-2 y}\right)+\tan ^{-1}\left(\frac{b-2 x}{a-2 y}\right)\right) \\
& +(2 x+b) \ln \left(\frac{(b+2 x)^{2}+(a+2 y)^{2}}{(b+2 x)^{2}+(a-2 y)^{2}}\right) \\
& \left.+(2 x-b) \ln \left(\frac{(b-2 x)^{2}+(a-2 y)^{2}}{(b-2 x)^{2}+(a+2 y)^{2}}\right)\right] \\
H_{y}=\frac{J}{8 \pi}[ & (4 x+2 b)\left(\tan ^{-1}\left(\frac{a+2 y}{b+2 x}\right)+\tan ^{-1}\left(\frac{a-2 y}{b+2 x}\right)\right) \\
& +(4 x-2 b)\left(\tan ^{-1}\left(\frac{a-2 y}{b-2 x}\right)+\tan ^{-1}\left(\frac{a+2 y}{b-2 x}\right)\right) \\
& +(2 y+a) \ln \left(\frac{(b+2 x)^{2}+(a+2 y)^{2}}{(b-2 x)^{2}+(a+2 y)^{2}}\right) \\
& \left.+(2 y-a) \ln \left(\frac{(b-2 x)^{2}+(a-2 y)^{2}}{(b+2 x)^{2}+(a-2 y)^{2}}\right)\right]
\end{aligned}
$$

The Equations (3) and (4), provide the analytical solutions for magnetic field components of a single rectangular busbar for the entire space. Furthermore, the magnetic field of any set of rectangular conductors, e.g. high-current busbars of an EAF transformer, can be calculated by applying the superposition principle and the use of the equations (3) and (4), considering the phase and the magnitude of the currents. In Figure 3, magnetic field distribution of a single busbar is presented. The center of the busbar is located at the origin of the coordinate system and it has dimensions of $150 \mathrm{~mm} \times 20 \mathrm{~mm}$ with $7 \mathrm{kA}$ current.

\section{Analytical Calculation of the Electromagnetic Force Between Two Busbars}

Using the analytical solution for the magnetic field of a single busbar, the quasi-static electromagnetic force on a conductor with the current density of $J$, can be calculated as follows [3]:

$$
F=J \times B
$$

where $F[N / m]$ is the electromagnetic force vector, $J\left[A / m^{2}\right]$ is the current density vector and $B[H]$ is the flux density vector which in this case equals to $\mu_{0} H$ where $\mu_{0}[H / m]$ is vacuum permeability 
75 and $H[A / m]$ is the magnetic field. By using (5) and assuming that the two adjacent busbars are 76 perpendicular to the $x-y$ plain, the differential force $d F$, on the volume element $d v$, can be expressed 77 as follows:

$$
d F=\mu_{0}\left(-J_{1} H_{y} a_{x}+J_{1} H_{x} a_{y}\right) d v
$$

$$
\begin{gathered}
F_{x}=-\mu_{0} J_{1} \int_{y_{c}-\frac{a}{2}}^{y_{c}+\frac{a}{2}} \int_{x_{c}-\frac{b}{2}}^{x_{c}+\frac{b}{2}} H_{y} d x d y \\
F_{y}=\mu_{0} J_{1} \int_{y_{c}-\frac{a}{2}}^{y_{c}+\frac{a}{2}} \int_{x_{c}-\frac{b}{2}}^{x_{c}+\frac{b}{2}} H_{x} d x d y
\end{gathered}
$$

Table 1: The electromagnetic force calculations for two sample busbars using both analytical solution and 2D FEA method.

\begin{tabular}{cccc}
\hline \hline Horizontal Distance (mm) & Analytical method (N/m) & 2D FEM (N/m) & Difference (\%) \\
\hline 50 & 24.50 & 24.48 & 0.10 \\
60 & 22.70 & 22.67 & 0.15 \\
70 & 21.11 & 21.09 & 0.12 \\
80 & 19.71 & 19.69 & 0.14 \\
90 & 18.47 & 18.44 & 0.16 \\
100 & 17.36 & 17.33 & 0.18 \\
110 & 16.36 & 16.33 & 0.21 \\
120 & 15.46 & 15.43 & 0.24 \\
130 & 14.65 & 14.62 & 0.25 \\
140 & 13.91 & 13.88 & 0.28 \\
150 & 13.24 & 13.20 & 0.30 \\
160 & 12.63 & 12.58 & 0.34 \\
170 & 12.06 & 12.02 & 0.37 \\
180 & 11.55 & 11.50 & 0.41 \\
190 & 11.07 & 11.02 & 0.45 \\
200 & 10.63 & 10.57 & 0.50 \\
210 & 10.22 & 10.16 & 0.54 \\
220 & 9.83 & 9.78 & 0.58 \\
230 & 9.48 & 9.42 & 0.63 \\
240 & 9.15 & 9.09 & 0.67 \\
250 & 8.84 & 8.78 & 0.72 \\
\hline
\end{tabular}




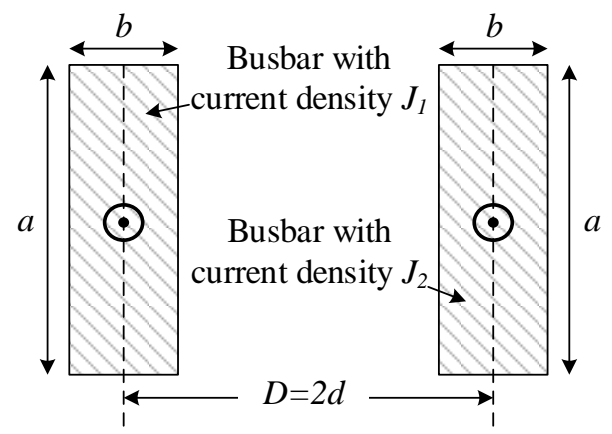

Figure 4: Two adjacent rectangular busbars with horizontal distance of $D$.

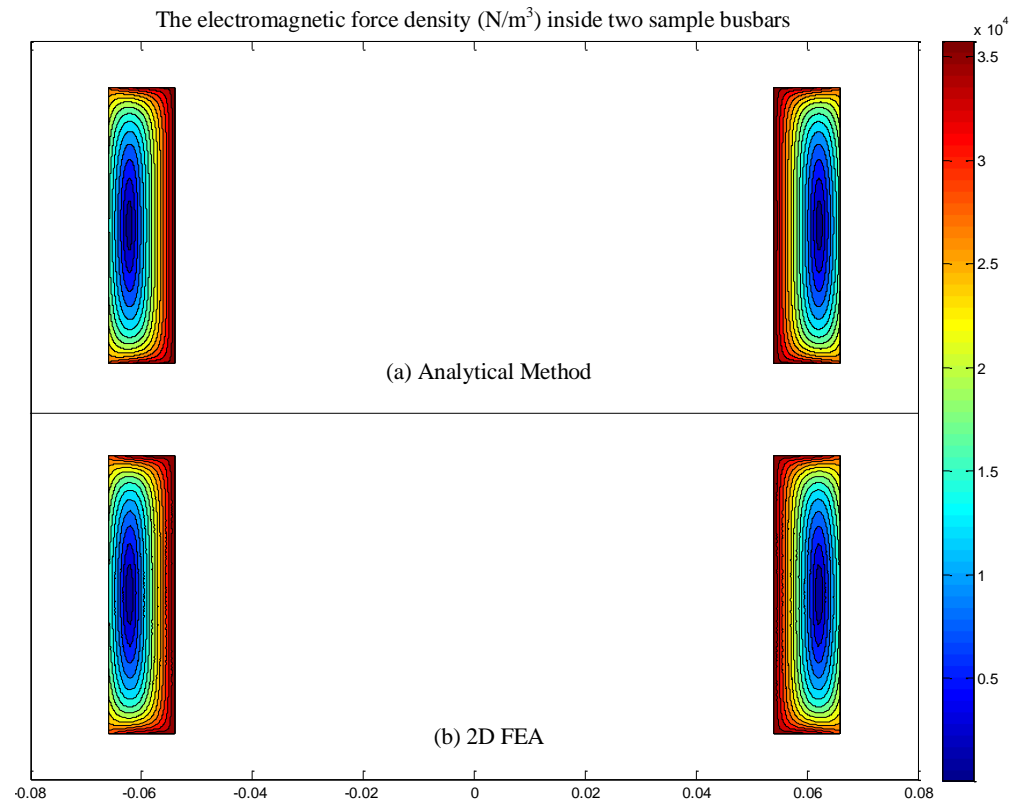

Figure 5: The force density inside rectangular busbars each carrying $5 \mathrm{kA}$ : a. Analytical method, b. 2D FEA using ANSYS.

It should be noted that (7) and (8) express the electromagnetic force components on the conductor in unit of length $[N / m]$. Equations (7) and (8) have very long analytical solutions which are not presented in this paper to save space. However, in the case where the two busbars are horizontally in-line as shown in Figure 4, due to the symmetry of the configuration, it can be concluded that the force component along $y$-axis is zero $\left(F_{y}=0\right)$. On the other hand, in this case, the root mean square 


$$
\begin{aligned}
F_{x}=\frac{\mu_{0} J_{1} J_{2} \cos (\theta)}{96 \pi}[ & 6 a(b-2 D)(b+2 D) \tan ^{-1}\left(\frac{a}{D}\right)-2 a\left(4 a^{2}-3 b^{2}\right) \tan ^{-1}\left(\frac{D}{a}\right) \\
& +a\left(4 a^{2}-3 b^{2}\right) \tan ^{-1}\left(\frac{D+b}{a}\right)+3 a(3 b-2 D)(b-2 D) \tan ^{-1}\left(\frac{a}{D-b}\right) \\
& +a\left(4 a^{2}-3 b^{2}\right) \tan ^{-1}\left(\frac{D-b}{a}\right)+3 a(3 b+2 D)(b-2 D) \tan ^{-1}\left(\frac{a}{D+b}\right) \\
& +4 D\left(3 a^{2}+D^{2}\right) \ln \left(D^{2}+a^{2}\right)-4(D+b)^{3} \ln \left(\frac{(D+b)^{2}+a^{2}}{D+b}\right) \\
& +4(D-b)^{3} \ln \left(\frac{(D-b)^{2}+a^{2}}{D-b}\right)+2(D+b)\left[(D+b)^{2}+3 a^{2}\right] \ln \left[(D+b)^{2}+a^{2}\right] \\
& \left.+2(D-b)\left[(D-b)^{2}+3 a^{2}\right] \ln \left[(D-b)^{2}+a^{2}\right]+8 D^{3} \ln \left(\frac{D^{2}+a^{2}}{D}\right)\right]
\end{aligned}
$$

where, $D$ is the distance between the centers of the busbars and $\theta$ is the phase difference between $J_{1}$ and $J_{2}$. Equation (9), is the analytical solution for the electromagnetic force between two rectangular busbars which is a function of the current densities and their phase difference, dimensions of the conductors and the distance between conductors. Superposition principle along with the presented analytical solutions can be applied for calculation of the total short circuit force on the high current busbars produced by adjacent busbars.

In order to verify the accuracy of the derived analytical solution for the calculation of the electromagnetic force between two current-carrying busbars, a simple configuration which consists of two busbars with dimensions of $20 \mathrm{~mm} \times 150 \mathrm{~mm}$, each carrying $5 \mathrm{kA}$ current is considered. By changing the distance between two busbars, from $50 \mathrm{~mm}$ to $250 \mathrm{~mm}$ with $10 \mathrm{~mm}$ steps, electromagnetic force is calculated using both analytical method and 2D FEA and the results are presented in Table 1. The ANSYS software is used for the FEA, and the models are set to have at least 50k elements to ensure that the electromagnetic force calculations have an acceptable accuracy. Infinite boundary conditions (which is called balloon boundary condition in the software) are used at the outer boundaries to model the space outside the drawing region as being infinitely large. It should be noted that in this case where the busbars are horizontally in-line, electromagnetic force in the direction of $y$-axis, is zero $\left(F_{y}=0\right)$. Table 1 , shows that the difference between two methods is less than $0.8 \%$ and therefore FEA verify the accuracy of the proposed method.

A computer with Intel Core i3 $41603.6 \mathrm{GHz}$ CPU and 4 GB of RAM is used for short-circuit calculations. Since the analytical solution for electromagnetic force is presented as a closed-form equation, the calculation time of the analytical solution is almost instantaneous but, the calculation time of any 2D FEA with minimum 50k elements takes about 30 seconds. In Figure 5, the electromagnetic force 
density inside the busbars with $100 \mathrm{~mm}$ distance, which are calculated using both analytical solution and FEA are shown. This figure shows that there is only a little discrepancy between the results of the analytical solution and the FEA.

As it is shown, the presented analytical solution for the electromagnetic force totally conform to the 2D FEA. Since the analytical solution presents the electromagnetic force as a closed-form equation, it can be used to provide a fast and efficient method by significantly reduction of calculation time.

\section{The Algorithm for the Calculation of Short Circuit Forces Based on the Theory of Images}

The method of images is a mathematical technique which has been widely used for the electromagnetic field problems with various boundary conditions. This method is used for simplification of the differential equations. The simplification is performed by extending the domain of the problem using the mirror images of the electromagnetic sources with respect to the symmetry planes or lines [2]. The symmetry planes (lines) are placed at the boundaries of the domain. As a result, complicated electromagnetic problems are simplified by replacing the boundary conditions with imaginary set of electromagnetic sources with specific magnitudes and locations. Therefore, the new model will lead to a simpler and more efficient solution for electromagnetic problem [18].

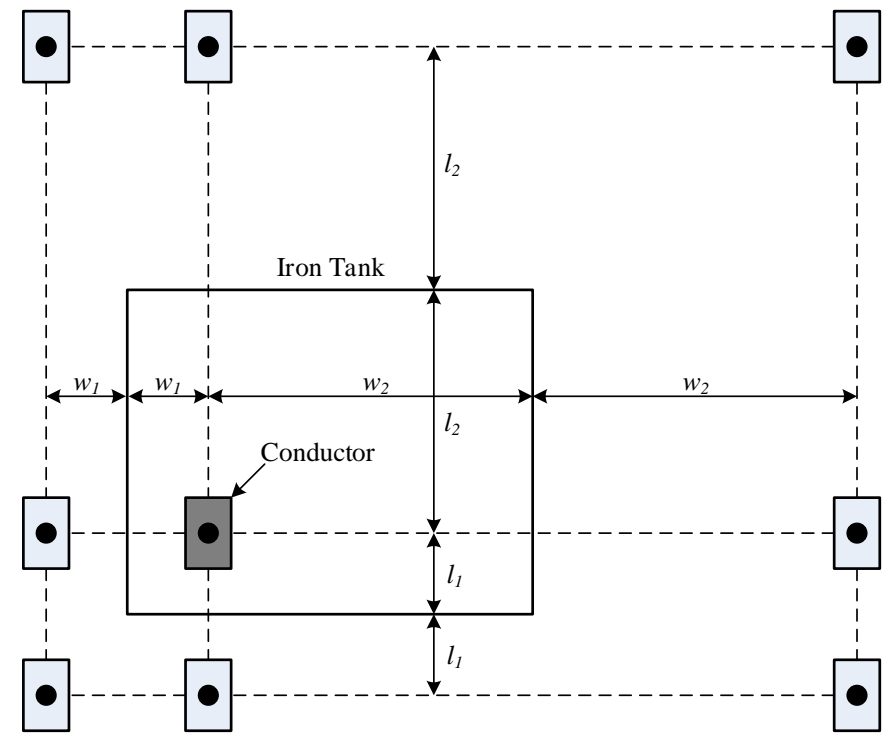

Figure 6: The first layer of images of a rectangular conductor with respect to four tank walls.

This method can be applied in all types of electromagnetic problems (static, quasi-static and transient) to simplify the calculation of magnetic field distribution of a current source in the vicinity of magnetic or electric conducting surfaces. The image current $I_{i}$ of a conductor, carrying current $I$, 
with respect to the boundary plane of a medium with a relative permeability of $\mu_{r}$ is given by the following equation $[6]$ :

$$
I_{i}=\frac{\mu_{r}-1}{\mu_{r}+1} I
$$

The method of images can be applied to the model of the busbars inside the tank walls of an EAF transformer, to replace the tank walls with the image currents. The image currents are mirrored with respect to four tank walls which are the symmetry planes. In Figure 6, a current carrying conductor inside an iron tank and its corresponding first layer of images are shown, where $w_{1}, w_{2}, l_{1}$ and $l_{2}$ represent the distance of the conductor to the left, right, up and down tank walls respectively. The location of the image currents with respect to tank walls can be determined using these four distances for each conductor. The new layers of images can be added to the model by applying the same principle to the previous layer images. Similarly, by applying the method of images and the superposition principle, the magnetic field distribution of any busbar configuration inside the tank walls can be calculated using (3) and (4). Also, the short circuit forces between any two real or imaginary rectangular busbar can be calculated analytically using (7) and (8). By applying the superposition principle to short forces between the busbar and other real and imaginary busbars, the short circuit force on each busbar can be calculated.

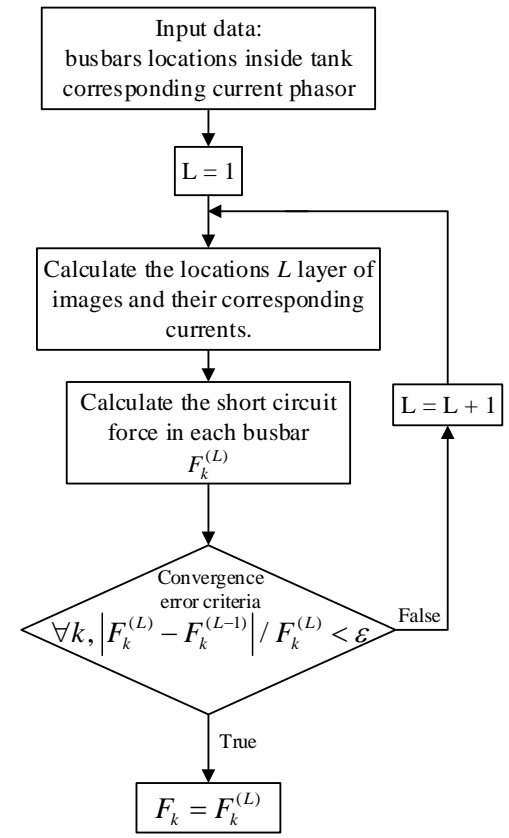

Figure 7: The flow chart of the iterative algorithm for the calculation of short circuit forces on the high-current busbars of EAF transformers.

The algorithm for the calculation of short circuit forces based on the theory of images is presented 
in Figure 7, where $L$ is the number of image layers, $k$ is the busbar number, $F_{k}$ is the short circuit force on the busbar $k$, and $F_{k}^{L}$ is the short circuit force calculated using $L$ layers of images. At the first step, the location and current magnitudes of the first layer of the images $(L=1)$ are calculated for all busbars, based on Figure 6 and equation (10). In every step of the algorithm electromagnetic force of each busbar $\left(F_{k}^{(L)}\right)$ is calculated and the relative error of the calculated forces is examined and new layers of images are added if the relative error is higher than desired error. and the locations of the new images and corresponding currents magnitude and phase is determined. The criteria for the convergence of the algorithm is defined as follows:

$$
\frac{\left|F_{k}^{(L)}-F_{k}^{(L-1)}\right|}{\left|F_{k}^{(L)}\right|}<\varepsilon
$$

where $\varepsilon$ is a predefined relative error. The algorithm continuous to a level at which the accuracy of the calculated forces for each busbar, reaches to an admissible value.

\section{Case Study Analysis}

A three-phase 30 MVA EAF transformer with 24 low voltage output busbars is considered as a case study. The specifications of the EAF transformer is presented in Table 2. This transformer has a peak three-phase short circuit current of $566 \mathrm{kA}$ on the low voltage side. Low voltage busbars have an open-delta connection and therefore each busbar will have $81.7 \mathrm{kA}$ short circuit current. A 2D diagram of the configuration of the busbars inside the tank walls and their corresponding dimensions and distances are shown in Figure 8. In this EAF transformer, each two adjacent busbars are jointed mechanically and electrically and therefore, the short circuit forces on the joint busbars are of interest. The busbars have dimensions of $12 \mathrm{~mm} \times 150 \mathrm{~mm}$ and the joint busbars have a $22 \mathrm{~mm}$ center to center distance.

Table 2: The specifications of the case study 30 MVA EAF transformer.

\begin{tabular}{lc}
\hline \hline Parameter & Value \\
\hline Rated power & $30 \mathrm{MVA}$ \\
Conversion ratio & $30.5 \mathrm{kV} / 0.498 \mathrm{kV}$ \\
LV nominal current & $49.1 \mathrm{kA}$ \\
Short circuit current & $566 \mathrm{kA}$ \\
No. of LV busbars & 24 \\
Busbar dimensions & $150 \mathrm{~mm} \times 12 \mathrm{~mm}$ \\
\hline
\end{tabular}




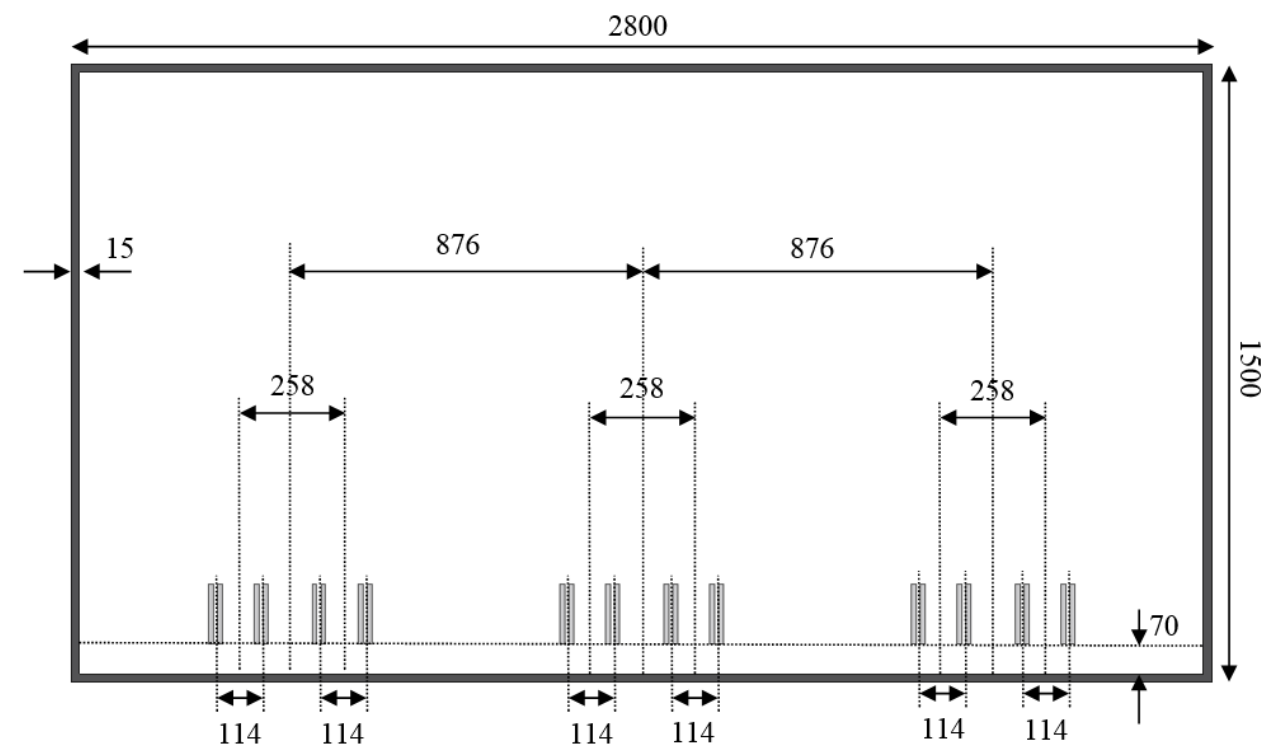

Figure 8: The configuration of busbars inside the tank walls of the 30 MVA case study EAF transformer. (The dimensions are in millimeters)

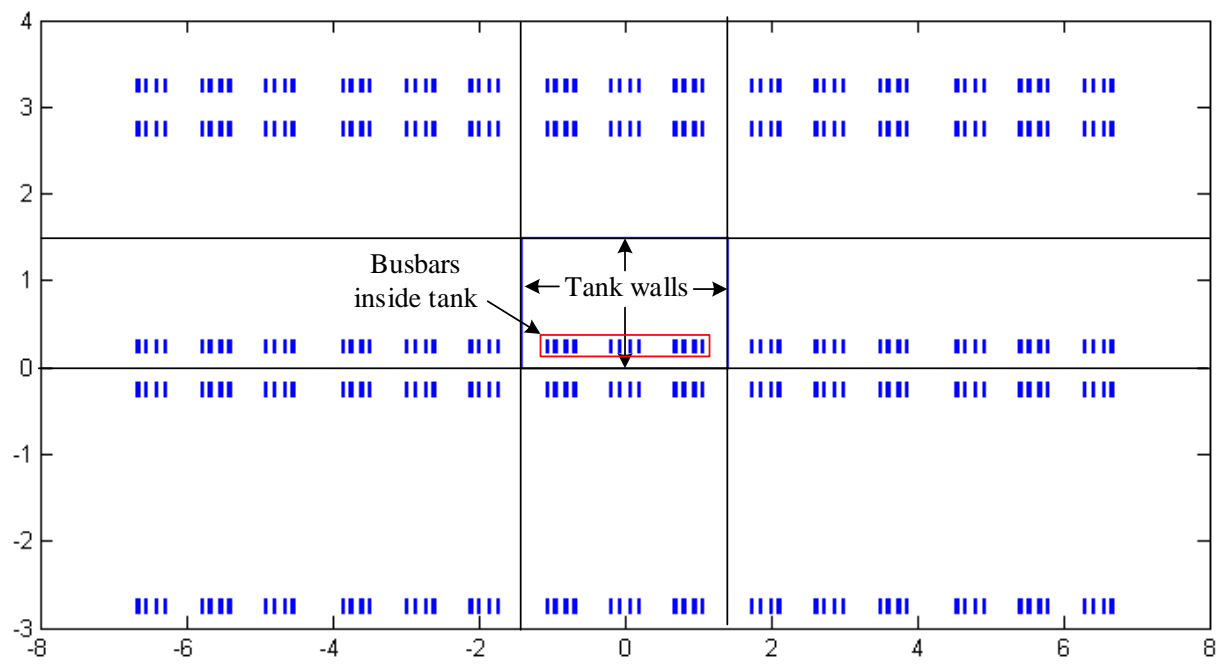

Figure 9: The busbars inside the tank walls of the 30 MVA case study transformer and their corresponding two layers of images at the outside the tank walls.

The short circuit forces on the busbars are calculated using both the proposed algorithm with maximum relative error of $0.03 \%$ and the and 2D FEA. The busbars with corresponding two layer of images are depicted in Figure 9.

The ANSYS software is used for the FEA, with 88,145 mesh elements which is shown in Figure 10. The material of the tank walls are defined as a steel with relative permeability of $\mu_{r}=550$. Also, 
infinite boundary conditions are used at the outer boundaries to model the space outside the wall tanks as being infinitely large. A computer with Intel Core i3 $41603.6 \mathrm{GHz}$ CPU and 4 GB of RAM is used for short-circuit calculations.

The calculation results are presented in Table 3. As it can be seen, the calculated short circuit forces using the proposed algorithm, completely conform with the calculations of 2D FEA results and the difference between two methods is less than $0.2 \%$. Also it should be noted that, there is a force in the direction of $y$-axis $\left(F_{y}\right)$, which is the impact of the iron tank walls on the busbars and as it is presented in Table 3 , its value is about $3 \%$ to $7 \%$ of the force component along $x$-axis $\left(F_{x}\right)$ in each busbar. Hence, this force component is only due to image currents outside the tank walls. In Figure 11, the convergence of the algorithm for the joint busbar no. 1, is shown. As it can be seen, the algorithm converges rapidly by increasing the number of image layers. This can be justified by knowing the fact that, as the number of image layer increases, the distance between the outer image layers and the busbars inside the tank walls increases rapidly, and therefore their effect on the short circuit forces on the busbars would be significantly low. Also, by comparing the calculation times which are presented in Table 3, it can be seen that the proposed algorithm has a much lower calculation time and it is 20 times faster than the 2D FEA. Furthermore, the presented calculations in Table 3 show that the short circuit forces on the high current busbars are extremely high and therefore should be considered as an important design measure while designing EAF transformers.

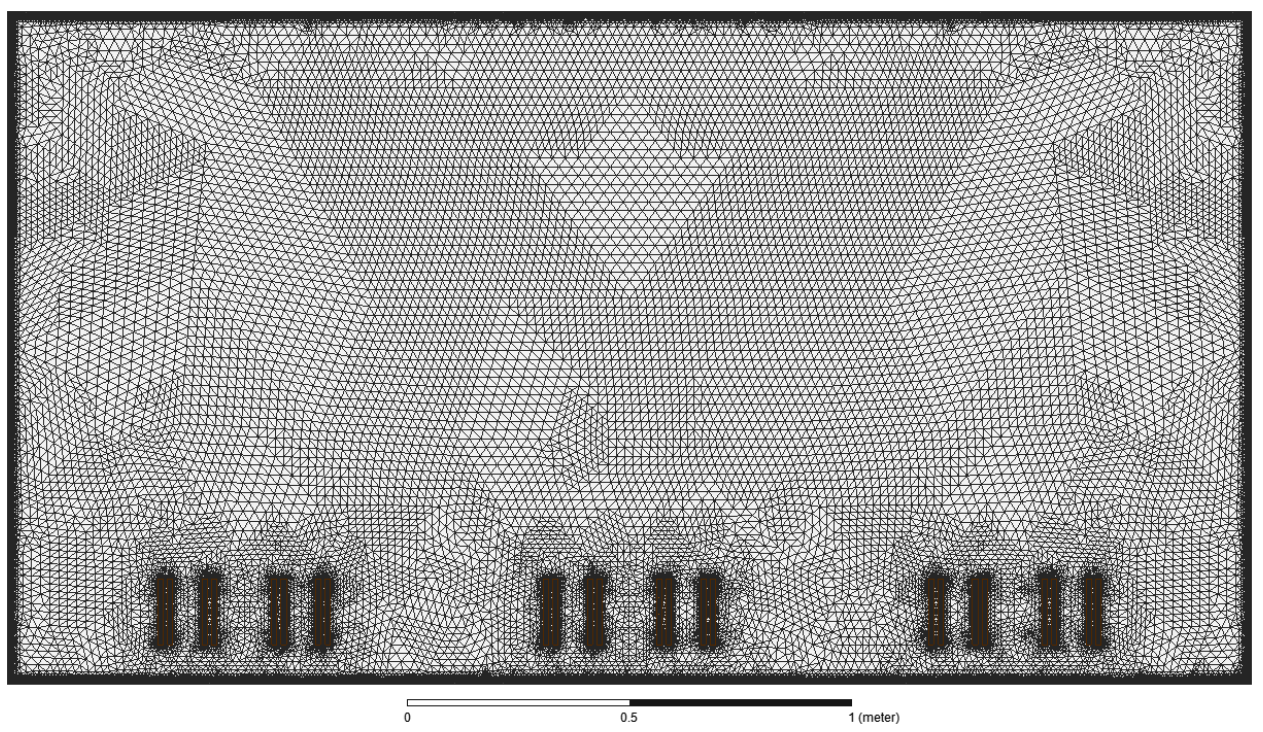

Figure 10: The mesh elements of the 2D finite element model consisting of 88,145 elements. 


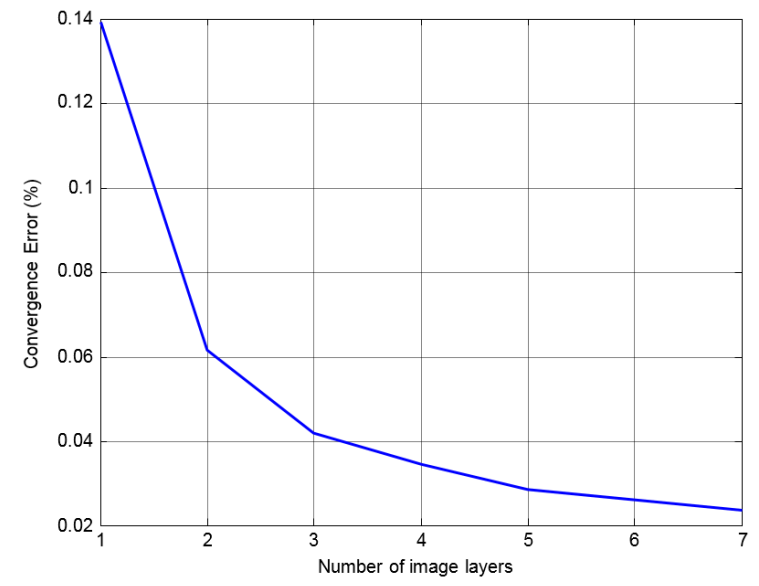

Figure 11: The convergence error vs. number of image layers for the joint busbars no. 1 in the 30 MVA EAF case study transformer.

Table 3: The results of the analytical method and FEM analysis of the case study transformer.

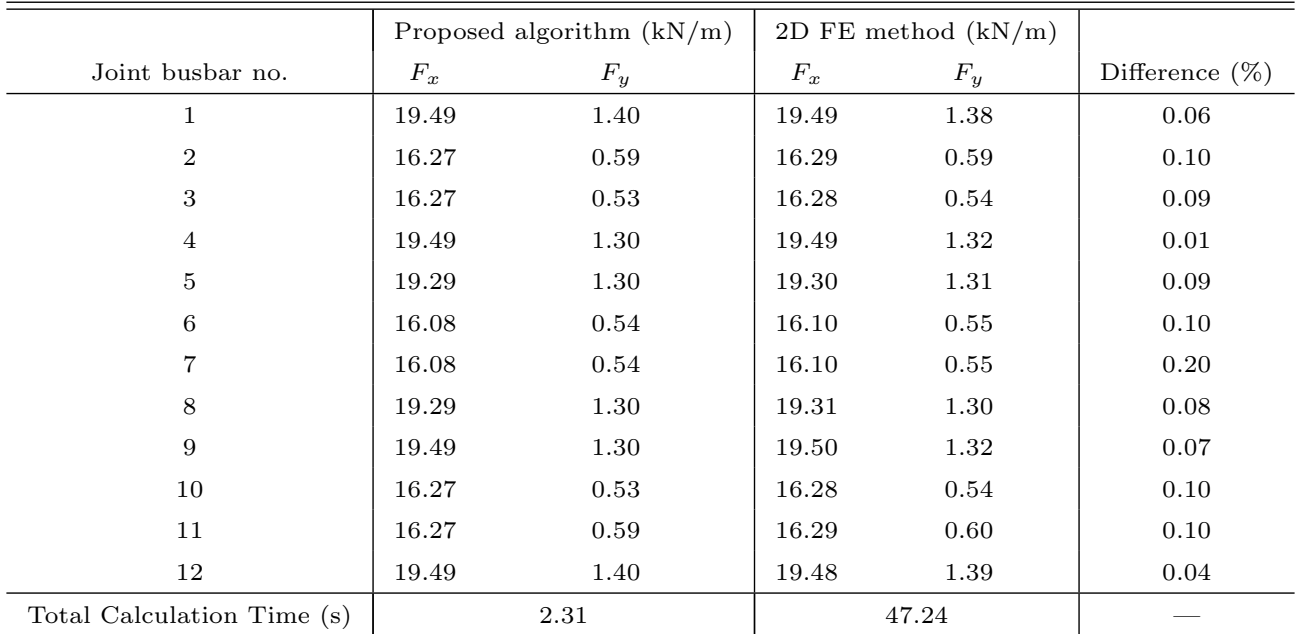

\section{Conclusion}

This paper has introduced an analytical solution for the electromagnetic force between rectangular busbars which is verified numerically using 2D FEA. The multi-layer method of images is then applied to the analytical solution to account for the effects of the tank walls on the busbars. An algorithm based on the multi-layer method of images is developed for the calculation of short circuit forces with the desired accuracy. Comparisons show that the difference between short circuit force calculation using the presented algorithm and 2D FEA is less than $0.2 \%$. Also, the presented algorithm is about 20 times faster than FEA with the same convergence error. Therefore, the proposed algorithm provides a fast and accurate method for the calculation of short circuit forces on the high current busbars of EAF 
transformers and as a result it can be used as a faster alternative for 2D FEA. Also, the calculations show that the short circuit forces on the high current busbars are very high and therefore should be considered as an important measure while designing EAF transformers.

\section{References}

[1] M. J. Heatcote, "The J and P Transformer Book," thirteenth edition, Elsevier Ltd, 2007.

[2] S. V. Kulkarni and S. A. Khaparde, "Transformer Engineering Design and Practice," Indian Institute of Technology, Marcel Dekker, Inc, 2004.

[3] R. M. Del Veccihio, B. poulin, P. T. Feghali, D. M. Shah, and R. ahuja, "Transformer design principles, with application to core form transformers," CRC press, 2010.

[4] K. Karsai, D. Kerenyi, and L. Kis, "Large power transformers," Elsevier Science Publishers, Amsterdam, Netherlands, 1987.

[5] W. H. Hayt and J. A. Buck, "Engineering electromagnetic," McGraw-Hill publication, sixth edition, 2001.

[6] D. K. Cheng, "Field and wave electromagnetics," Addison-Wesley, 2nd edition, January, 1989.

[7] A. G. Kladas, M. P. Papadopoulos, and J. A. Tegopoulos, "Leakage flux and force calculation on power transformer windings under short-circuit: 2D and 3D models based on the theory of images and the finite element method compared to measurements," IEEE Transactions on Magnetics, Vol. 30, Issue 5, Part 2, 1994.

[8] M. Ardebili, K. Abbaszadeh, S. Jamali, and H. A. Toliyat, "Winding Arrangement Effects on Electromagnetic Forces and Short-Circuit Reactance Calculation in Power Transformers via Numerical and Analytical Methods," 2006 12th Biennial IEEE Conference on Electromagnetic Field Computation, pp. 506, 2006.

[9] Ahn Hyun-Mo, Lee Ji-Yeon, Kim Joong-Kyoung, Oh Yeon-Ho, Jung Sang-Yong, and Hahn SungChin, "Finite-Element Analysis of Short-Circuit Electromagnetic force in Power Transformer," IEEE Transactions on Industry Applications, vol. 47, Issue 3, 2011.

[10] L. Strac, F. Kelemen, and D. Zarko, "Analysis of short-circuit forces at the top of the low voltage U-type and I-type winding in a power transformer," EPE-PEMC 2008, 13th Power Electronics and Motion Control Conference, pp. 855-858, 2008. 
[11] M. Steurer and K. Frohlich, "The impact of inrush currents on the mechanical stress of high voltage power transformer coils," IEEE Transactions on Power Delivery, vol. 17, No. 1, January 2002.

[12] G. B. Kumbhar and S. V. Kulkarni, "Analysis of Short-Circuit Performance of Split-Winding Transformer Using Coupled Field-Circuit Approach," IEEE Transactions on Power Delivery, vol. 22, Issue 2, pp. 936-943, 2007.

[13] V. Nabaei, S. A. Mousavi, K. Miralikhani, and H. Mohseni, "Balancing Current Distribution in Parallel Windings of Furnace Transformers Using the Genetic Algorithm," IEEE Transactions on Magnetics, Volume 46 , Issue 2, 2010.

[14] B. Stojcic and D. Miljavec, "Current distribution in the low-voltage winding of the furnace transformer," International Journal of Electrical Power and Energy Systems, vol. 43, Issue 1, pp. 12511258, Dec. 2012.

[15] D. G. Triantafyllidis, P. S. Dokopoulos, and D. P. Labridis, "Parametric short-circuit force analysis of three-phase busbars-a fully automated finite element approach," IEEE Transactions on Power Delivery, vol.18, no. 2, pp. 531-537, Apr. 2003.

[16] B. Shen, X. Zhang, and D. Zhou, "Analysis and calculation of short-circuit electro-dynamic forces on rectangular bus bars," in Power Electronics and Motion Control Conference, 2009. IPEMC '09. IEEE 6th International, vol., no., pp. 2618-2621, May 2009.

[17] Krakowski, M. R. Kazmierski, and I. M. Kersz, "Effect of current distribution in parallel bars on electromagnetic field at nearby steel wall," Electric Power Applications, IEE Proceedings B, Volume 136, pp. 161167, 1989.

[18] P. Gomez and F. de Leon, "Accurate and efficient computation of the inductance matrix of transformer windings for the simulation of very fast transients," IEEE Trans. Power Del., vol. 26, pp. 1423-1431, Jul. 2011.

[19] J. C. Olivares, R. Escarela-Perez, S. V. Kulkarni, F. de Leon, and M. A. Venegas-Vega, "2D finiteelement determination of tank wall losses in pad-mounted transformers," Electric Power Systems Research 71, pp. 179-185, 2004.

[20] S. V. Kulkarni and S. A. Khaparde, "Stray Loss Evaluation in Power Transformers - A Review," IEEE Power Engineering Society Winter Meeting, Vol. 3, pp. 2269-2274, 2000. 
[21] E. J. Tuohy, "Computation of Eddy Currents, Shielding and Transient Forces in Power System Equipment by the Method of Images," IEEE Transactions on Power Apparatus and Systems, Volume PAS-90, Issue 3, pp. 1271-1277, 1971.

[22] M. Lambert, F. Sirois, M. Martinez-Duro, J. Mahseredjian, "Analytical Calculation of Leakage Inductance for Low-Frequency Transformer Modeling," IEEE Transactions on Power Delivery, vol. 28, no. 1, pp. 507-515, Jan. 2013.

[23] B. M. Ebrahimi, A. Fereidunian, S. Saffari and J. Faiz, "Analytical estimation of short circuit axial and radial forces on power transformers windings," IET in Generation, Transmission and Distribution, vol. 8, no. 2, pp. 250-260, Feb. 2014.

[24] Hyun-Mo Ahn, Yeon-Ho Oh, Joong-Kyoung Kim, Jae-Sung Song and Sung-Chin Hahn, "Experimental verification and finite element analysis of short-circuit electromagnetic force for dry-type transformer," IEEE Transactions on Magnetics, vol. 48, no. 2, pp. 819-822, Feb. 2012. 